\title{
On the Cauchy Problem for Systems of Conservation Laws
}

\author{
Alberto Bressan*
}

\section{Résumé}

This paper contains an informal discussion of recent results concerning the well posedness of the Cauchy problem for a nonlinear system of conservation laws in one space dimension. The system is assumed to be strictly hyperbolic, with each characteristic field either linearly degenerate or genuinely nonlinear.

\section{The semigroup approach}

This paper contains an informal discussion of recent results concerning the well posedness of the Cauchy problem for a nonlinear $n \times n$ system of conservation laws in one space dimension:

$$
\begin{gathered}
u_{t}+[F(u)]_{x}=0, \\
u(0, x)=\bar{u}(x) .
\end{gathered}
$$

The system is assumed to be strictly hyperbolic, with each characteristic field either linearly degenerate or genuinely nonlinear [23, 28]. In this framework, a basic existence problem can be formulated as follows.

(EP1) Show that there exists some (nontrivial) domain $\mathcal{D} \subset \mathbf{L}^{1}$ such that, for every initial data $\bar{u} \in \mathcal{D}$, the Cauchy problem (1)-(2) has at least one global, entropy-admissible, weak solution $u:[0, \infty[\mapsto \mathcal{D}$.

We recall that, for smooth initial data $\bar{u}$, the local existence of a solution is well known [27]. Due to genuine nonlinearity, however, this solution may lose its regularity. Indeed, its gradient $u_{x}$ may become infinite within finite time [21]. For this reason, in general, solutions can be constructed globally in time only within a space of discontinuous functions. A natural choice is the space BV of integrable functions $u: \mathbb{R} \mapsto \mathbb{R}^{n}$ with bounded variation. The existence problem (EP1) was solved in the fundamental paper of Glimm [20], on a domain $\mathcal{D}$ consisting of functions with suitably small total variation. For future reference, we recall the construction of the positively invariant domain $\mathcal{D}$. Consider a piecewise constant function $u$, say with jumps at the points $x_{1}<\cdots<x_{N}$. For $\alpha=1, \ldots, N$, call $\sigma_{\alpha}^{1}, \ldots, \sigma_{\alpha}^{n}$ the strengths of the waves in the standard self-similar solution of the Riemann problem with data $u\left(x_{\alpha}-\right)$, $u\left(x_{\alpha}+\right)$. The total strength of waves in $u$ and the potential for future wave interactions are defined respectively as

$$
V(u) \doteq \sum_{\alpha=1}^{N} \sum_{i=1}^{n}\left|\sigma_{\alpha}^{i}\right|, \quad Q(u) \doteq \sum_{\mathcal{A}}\left|\sigma_{\alpha}^{i} \sigma_{\beta}^{j}\right|,
$$

*S.I.S.S.A., Via Beirut 4, Trieste 34014 Italy. 
where the second sum ranges over all couples of approaching waves. One then defines

$$
\mathcal{D} \doteq c l\left\{u \in \mathbf{L}^{1}\left(\mathbb{R} ; \mathbb{R}^{n}\right), \quad u \text { is piecewise constant, } V(u)+\kappa_{0} Q(u)<\delta_{0}\right\},
$$

for suitable constants $\kappa_{0}, \delta_{0}>0$, where $c l$ denotes closure.

Concerning uniqueness, we remark that, for smooth solutions, (1) is equivalent to the quasilinear system

$$
u_{t}+A(u) u_{x}=0 .
$$

Here $A(u)=D F(u)$ is the Jacobian matrix of $F$ at $u$. If $u, v$ are both solutions of (1), their difference $w=u-v$ satisfies

$$
w_{t}+A(u(t, x)) w_{x}+[D A(t, x) \cdot w] v_{x}(t, x)=0,
$$

with

$$
D A(t, x) \doteq \int_{0}^{1} D A(\theta u(t, x)+(1-\theta) v(t, x)) d \theta .
$$

Observe that (6) is a linear homogeneous hyperbolic system for $w$, with coefficients depending on $t, x$. If the functions $u, v$ are Lipschitz continuous, then these coefficients have some degree of regularity, which allows one to derive a priori estimates on the norm of $w$. In particular, from $w(0)=0$ we deduce $w(t)=0$ for all $t>0$, hence $u \equiv v$, proving uniqueness.

If the solutions $u, v$ are discontinuous, however, the above approach is no longer valid, and a proof of uniqueness becomes considerably more difficult. Progress in this direction has been achieved recently by a new approach, based on the preliminary construction of a semigroup of solutions of (1). Instead of tackling the uniqueness question directly, it is convenient to consider first a more comprehensive existence problem.

(EP2) Show that there exists a (nontrivial) domain $\mathcal{D} \subset \mathbf{L}^{1}$ and a continuous flow $S$ : $\mathcal{D} \times[0, \infty[\mapsto \mathcal{D}$ with the properties:

(i) $S_{0} u=u, \quad S_{s} S_{t} u=S_{s+t} u$,

(ii) $\left\|S_{t} u-S_{s} v\right\| \leq L \cdot(|t-s|+\|u-v\|)$,

(iii) every trajectory $t \mapsto S_{t} u$ is a weak, entropy-admissible solution of the partial differential equation (1).

Observe that (i) is the semigroup property, while (ii) states that the flow is globally Lipschitz continuous, both with respect to time and to the initial data.

The main difference between the problems (EP1) and (EP2) is that, in the latter, we wish to construct solutions simultaneously for all $\bar{u} \in \mathcal{D}$, continuously depending on the initial data. Establishing the existence of a semigroup $S$ satisfying (i)-(iii) is thus a more difficult task than proving the existence of one single solution. This was achieved in [9] for $2 \times 2$ systems, and in [11] for general $n \times n$ systems. More precisely, we have

Theorem 1. Let the system (1) be strictly hyperbolic in a neighborhood of the origin, and assume that each characteristic field is either linearly degenerate or genuinely nonlinear. Then there exist a domain $\mathcal{D}$ as in (4) and a continuous semigroup $S: \mathcal{D} \times[0, \infty[\mapsto \mathcal{D}$ satisfying the above conditions (i)-(iii).

The uniqueness of the semigroup, on the other hand, can be proved rather easily. In this connection, it is convenient to introduce a new condition, stating that the flow is consistent with the standard solutions of the Riemann problem: 
(iv) If $\bar{u} \in \mathcal{D}$ is piecewise constant, then for all $t>0$ sufficiently small the function $u(t, \cdot)=$ $S_{t} \bar{u}$ coincides with the solution of (1)-(2) obtained by piecing together the standard self-similar solutions of the Riemann problems determined by the jumps in $\bar{u}$.

One of the main results in [7] states that, for a given domain $\mathcal{D}$ of the form (4), there can exists at most one semigroup $S: \mathcal{D} \times[0, \infty[\mapsto \mathcal{D}$ satisfying (i), (ii) and (iv). In the positive case, the condition (iii) is also satisfied. We then call $S$ a Standard Riemann Semigroup generated by the system (1).

Having established the existence and uniqueness of the semigroup, we are in a much better position to study the uniqueness of the solution to a particular Cauchy problem, and its dependence on the initial data. Indeed, the analysis relies on the following simple lemma.

Lemma 1. Let $S: \mathcal{D} \times[0, \infty[\mapsto \mathcal{D}$ be a continuous flow satisfying the properties (i)-(ii) in (EP2). For every Lipschitz continuous map $w:[0, T] \mapsto \mathcal{D}$ one then has the estimate

$$
\left\|w(T)-S_{T} w(0)\right\|_{\mathrm{L}^{1}} \leq L \cdot \int_{0}^{T}\left\{\liminf _{h \rightarrow 0+} \frac{\left\|w(t+h)-S_{h} w(t)\right\|_{\mathrm{L}^{1}}}{h}\right\} d t .
$$

A proof will be given in Section 6.

Observe that the only relevant assumption of the lemma is the Lipschitz continuity of the Semigroup. No reference is here made to the particular equation (1). Our main application, however, will be in connection with the Cauchy problem (1)-(2). Let $w=w(t)$ be any approximate solution, Lipschitz continuous as a function of time, taking the correct initial condition $w(0)=\bar{u}$. If (iii) holds, the exact solution is then provided by the function $t \mapsto S_{t} \bar{u}$. At time $T$, the $\mathbf{L}^{1}$ distance between the approximate and the exact solution is estimated by (7). Observe that the integrand in (7) determines the instantaneous error rate. The estimate thus states that these local errors are magnified at most by a factor of $L$, and summed up over the interval $[0, T]$. The formula (7) has two main applications.

1. Let $u=u(t, x)$ be a weak solution of (1), Lipschitz continuous as a function of time with values in $\mathbf{L}^{1}$. If one can show that

$$
\liminf _{h \rightarrow 0+} \frac{\left\|w(t+h)-S_{h} w(t)\right\|_{\mathbf{L}^{1}}}{h}=0 \quad \text { for a.e. } t>0,
$$

then one can conclude that $w(t)=S_{t} w(0)$ for all $t$. Since we already know that the semigroup $S$ is unique, this yields a new method for proving uniqueness of solutions to a given Cauchy problem. This approach was pursued in [7], establishing the uniqueness of limit solutions obtained by the Glimm scheme [20,24] or by wave-front tracking approximations [2, 5, 19, 26]. Using similar ideas, a more general uniqueness result for entropy-weak solutions was proved in [13], provided that the total variation of $u$ along segments in the $t$ - $x$ plane can be suitably controlled.

2. Let $w=w(t, x)$ be an approximate solution of (1) constructed by a wave-front tracking method. For every $T>0$, the distance between $w(T)$ and the exact solution $S_{T} w(0)$ can then be estimated by computing the integrand in (7). More precisely, assume that at a generic time $t$ the function $w$ is piecewise constant, with jumps located at points $x_{1}(t)<\cdots<x_{N}(t)$, travelling with speed $\dot{x}_{\alpha}, \alpha=1, \ldots, N$. Call $\omega_{\alpha}=\omega_{\alpha}(t, x)$ the self-similar solution of the Riemann problem with data $w\left(t, x_{\alpha}-\right), w\left(t, x_{\alpha}+\right)$. Letting $\hat{\lambda}$ be an upper bound for all wave 
speeds and choosing $h_{0}>0$ suitably small, we can write

$$
\lim _{h \rightarrow 0+} \frac{\left\|w(t+h)-S_{h} w(t)\right\|_{\mathbf{L}^{1}}}{h}=\sum_{\alpha=1}^{N} \frac{1}{h} \int_{-\hat{\lambda} h_{0}}^{\hat{\lambda} h_{0}}\left|w\left(t+h_{0}, x_{\alpha}(t)+y\right)-\omega_{\alpha}\left(h_{0}, y\right)\right| d y
$$

In other words, the instantaneous error rate in (9) is measured by the differences between the travelling fronts of $w$ and the exact self-similar solutions of the corresponding Riemann problems. This provides an explicitly computable error bound for the wave-front tracking method [7]. By a more refined analysis, error estimates for the Glimm scheme were obtained in $[15]$.

\section{Examples of contractive metrics}

By the remarks in the previous section, in order to establish the well posedness of the Cauchy problem (1)-(2), a key step is the construction of a Lipschitz semigroup of solutions. Toward this goal, one needs to construct a sequence of approximations $u_{\nu}=u_{\nu}(t, x)$, carefully controlling:

(1) The total variation Tot.Var. $\left(u_{\nu}(t, \cdot)\right)$ of each approximate solution.

(2) The distance $\left\|u_{\nu}(t, \cdot)-v_{\nu}(t, \cdot)\right\|_{\mathrm{L}^{1}}$ between any two approximate solutions.

For small BV initial data, the Glimm scheme [20, 24] and the wave-front tracking algorithms $[2,5,19,26]$ provide control of the total variation, in terms of a wave interaction potential. Unfortunately, none of the algorithms found in the standard literature yields estimates on the dependence on the initial data. In particular, all convergence proofs are based on a compactness argument, which does not provide informations on the uniqueness of the limit. New constructive algorithms must therefore be devised.

A useful technique for estimating the distance between solutions is the introduction of a new distance $d_{*}$, which is equivalent to the old distance and nonexpansive w.r.t. the flow generated by (1). In connection with hyperbolic systems, this approach was first introduced in [4], then extended in $[9,11]$ to more general systems. To familiarize the reader with this method, we collect here a few simple examples of dynamical systems

$$
\frac{d}{d t} u(t)=\Phi(u(t))
$$

whose flow $(\bar{u}, t) \mapsto S_{t} \bar{u}$ is non-expansive for a suitable distance $d_{*}$.

A well known class of evolution problems generating a contractive semigroup are those of the form

$$
0 \in \frac{d u}{d t}+B(u)
$$

where $B$ is a hyperaccretive operator on a Banach space $E$ (see Chapter 3 in [18] for details). Scalar conservation laws, even in several space dimensions, fit into this framework [17, 22]. In this case, the flow is contractive w.r.t. the standard distance $d(u, v)=\|u-v\|$. Below, we consider situations where the flow is contractive not for $d$, but only for some equivalent distance $d_{*}$

In some easy cases, a contractive distance can be defined explicitly. 
Example 1. On the domain $\mathcal{D} \doteq] 0, \infty[$, consider the differential equation

$$
\dot{x}=c x .
$$

Of course, the corresponding flow is $S_{t} x=e^{c t} x$. The Euclidean distance $\left|S_{t} u-S_{t} v\right|$ between two solutions thus increases in time. However, one easily checks that this flow is non-expansive w.r.t. the distance

$$
d_{*}(x, y) \doteq|\log (x / y)|
$$

Example 2. Let $\phi$ be a smooth scalar function satisfying $C^{-1} \leq \phi(x) \leq C$ for some constant $C>1$. Then the evolution equation

$$
u_{t}+\phi(x) u_{x}=0
$$

generates a linear semigroup on the space $\mathbf{L}^{1}$, which is non-expansive for the distance

$$
d_{*}(u, v) \doteq \int \frac{|u(x)-v(x)|}{\phi(x)} d x .
$$

By the assumptions on $\phi$, the above $d_{*}$ is uniformly equivalent to the standard $\mathbf{L}^{1}$ distance.

In other cases, the distance is defined in terms of a Riemann-type metric, taking the point of view of differential geometry. More precisely, let $\mathcal{D}$ be a closed domain in a Banach space $E$. The construction of a metric $d_{*}$ on $\mathcal{D}$ will involve:

- A dense subset $\mathcal{D}^{\prime} \subset \mathcal{D}$.

- At each point $u \in \mathcal{D}^{\prime}$, a space $T_{u}$ of tangent vectors, equipped with a weighted norm $\|\mathbf{v}\|_{u}$.

- A family $\mathcal{R} \mathcal{P}$ of suitably regular paths $\gamma:[a, b] \mapsto \mathcal{D}^{\prime}$.

We assume that the family $\mathcal{R P}$ is closed under concatenation of paths. Moreover, for each $\gamma \in \mathcal{R} \mathcal{P}$, we require that the differential $\mathbf{v}=D \gamma=d \gamma(\theta) / d \theta$ is a well defined tangent vector in $T_{\gamma(\theta)}$, for almost every $\theta \in[a, b]$. The weighted length of a regular path $\gamma$ can thus be defined as

$$
\|\gamma\|_{*} \doteq \int_{a}^{b}\|D \gamma(\theta)\|_{\gamma(\theta)} d \theta
$$

Let now $u, w \in \mathcal{D}^{\prime}$. Call $\Sigma_{u, w}$ the family of all paths $\gamma \in \mathcal{R P}$ joining $u$ with $w$. By setting

$$
d_{*}(u, w) \doteq \inf \left\{\|\gamma\|_{*}, \quad \gamma \in \Sigma_{u, w}\right\},
$$

one defines a distance on the dense subset $\mathcal{D}^{\prime} \subset \mathcal{D}$. By continuity, $d_{*}$ can then be extended to the whole domain $\mathcal{D}$.

At this stage, the relevant question is whether the metric $d_{*}$ is non-expansive in connection with the flow of (10). Clearly, this is the case provided that, for every $\gamma \in \mathcal{R P}$, the weighted length of the path $S_{t} \gamma: \theta \mapsto S_{t}(\gamma(\theta))$ is a non-increasing function of time. To check this condition, for each solution $u$ of (10) we consider the corresponding linearized evolution equation for tangent vectors

$$
\frac{d}{d t} \mathbf{v}(t)=D \Phi(u(t)) \cdot \mathbf{v}(t) .
$$

A natural assumption now is: 
(A1) For every solution $u=u(t)$ of (10) taking values inside $\mathcal{D}^{\prime}$, and every solution $\mathbf{v}=\mathbf{v}(t)$ of the variational equation (16), the weighted norm of the tangent vector

$$
t \mapsto\|\mathbf{v}(t)\|_{u(t)}
$$

is a non-increasing function of time.

Condition (A1) is "almost sufficient" for the contractivity of the flow of (10). A formal proof goes as follows. Let $\bar{u}, \bar{w} \in \mathcal{D}^{\prime}$ and $\varepsilon>0$ be given. Call $u(t) \doteq S_{t} \bar{u}, w(t) \doteq S_{t} \bar{w}$ the trajectories of (10) with initial data $\bar{u}, \bar{w}$, respectively. Choose a regular path $\gamma:[a, b] \mapsto \mathcal{D}^{\prime}$ joining $\bar{u}$ with $\bar{w}$, such that

$$
\|\gamma\|_{*} \leq d_{*}(\bar{u}, \bar{w})+\varepsilon
$$

Define

$$
u^{\theta}(t) \doteq S_{t}(\gamma(\theta)), \quad \mathbf{v}^{\theta}(t) \doteq \frac{\partial}{\partial \theta} S_{t}(\gamma(\theta)) .
$$

Each $u^{\theta}$ is thus a solution of (10), while $\mathbf{v}^{\theta}$ solves the corresponding linearized variational equation (16). Using (A1) we now compute

$$
\begin{aligned}
d_{*}(u(t), w(t)) & \leq\left\|S_{t} \gamma\right\|_{*} \\
& =\int_{a}^{b}\left\|\mathbf{v}^{\theta}(t)\right\|_{u^{\theta}(t)} d \theta \\
& \leq \int_{a}^{b}\left\|\mathbf{v}^{\theta}(0)\right\|_{u^{\theta}(0)} d \theta \\
& \leq d_{*}(\bar{u}, \bar{w})+\varepsilon .
\end{aligned}
$$

Since $\varepsilon>0$ was arbitrary, this achieves the proof. In the previous argument, we tacitly assumed that the regularity of every path is preserved by the flow:

(A2) For every $\gamma \in \mathcal{R} \mathcal{P}$ and every $t>0$, the path $S_{t} \gamma$ still belongs to the family $\mathcal{R P}$ of regular paths.

In many cases involving finite dimensional evolution equations, the above assumption is trivially satisfied. In connection with the system of conservation laws (1), however, (A2) fails, due to the possible loss of regularity in piecewise Lipschitz solutions. This technical difficulty will be addressed in Section 4.

We conclude this section with two more examples of flows which are contractive w.r.t. a Riemann-type distance.

Example 3. For the evolution $\dot{x}=c x$ considered in Example 1, introduce the Riemann metric

$$
\|\mathbf{v}\|_{x} \doteq \frac{1}{x} \cdot|\mathbf{v}|
$$

Along any solution $x=x(t)$ of (12), we now have

$$
\dot{\mathbf{v}}=c \mathbf{v}, \quad \frac{d}{d t}\|\mathbf{v}(t)\|_{x(t)}=0 .
$$

It is thus clear that the corresponding distance

$$
d_{*}(x, y)=\left|\int_{x}^{y} \frac{1}{s} d s\right| \quad x, y>0,
$$

is non-expansive for the flow of (12). Actually, we have just carried out an alternative construction of the same distance $d_{*}$ in (13).

ESAIM: Proc., VoL. 3, 1998, 23-36 
Example 4. On the space $E=\mathbf{L}^{1}([0,1] ; \mathbb{R}) \times \mathbf{L}^{1}([0,1] ; \mathbb{R})$, consider the discontinuous evolution equation

$$
\left\{\begin{array}{l}
u_{t}(x)=\phi(\operatorname{meas}\{y ; v(y)<u(x)\}), \\
v_{t}(y)=\psi(\operatorname{meas}\{x ; u(x)>v(y)\}),
\end{array}\right.
$$

where $\phi, \psi$ are smooth functions satisfying

$$
\phi(s)>1, \quad \psi(s)<-1, \quad\left|\frac{d \phi}{d s}\right|<\kappa, \quad\left|\frac{d \phi}{d s}\right|<\kappa \quad \forall s \in[0,1],
$$

for some constant $\kappa>0$. The corresponding flow is then contractive w.r.t. the weighted distance $d_{*}$ on $E$, defined in terms of the Riemannian metric

$$
\begin{aligned}
\|(\mathbf{w}, \mathbf{z})\|_{(u, v)} \doteq \quad & \int_{0}^{1}|\mathbf{w}(x)| \cdot \exp (-\kappa \cdot \operatorname{meas}\{y ; v(y)<u(x)\}) d x \\
& +\int_{0}^{1}|\mathbf{z}(y)| \cdot \exp (-\kappa \cdot \operatorname{meas}\{x ; u(x)<v(y)\}) d x .
\end{aligned}
$$

It is interesting to observe that, if $u, v$ solve (20), then the two functions

$$
U(t, \xi) \doteq \operatorname{meas}\{x ; u(t, x)>\xi\}, \quad V(t, \xi) \doteq \operatorname{meas}\{x ; v(t, y)<\xi\},
$$

provide a solution to the linearly degenerate, strictly hyperbolic system

$$
U_{t}+\phi(V) U_{\xi}=0, \quad V_{t}+\psi(U) V_{\xi}=0 .
$$

Another example of a Riemann-type metric on $\mathbb{R}^{n}$, contractive for the flow of a discontinuous differential equation, can be found in [8].

\section{A contractive metric for hyperbolic systems}

In this section we outline the consruction of a distance, equivalent to the $\mathbf{L}^{1}$ metric, which is contractive w.r.t. the flow generated by a system of conservation laws.

Consider the domain $\mathcal{D}$ of functions with small total variation, defined as in (4). Let $\mathcal{D}_{P L}$ be the dense subset of Piecewise Lipschitz functions.

We recall below the definition of generalized differential of a path $\gamma:[a, b] \mapsto \mathbf{L}^{1}$, introduced in [14]. For any $u \in \mathbf{L}^{1}$, on the family $\Sigma_{u}$ of all continuous paths $\gamma:\left[0, \theta_{0}\right] \mapsto \mathbf{L}^{1}$ such that $\gamma(0)=u$, consider the equivalence relation

$$
\gamma \sim \gamma^{\prime} \quad \text { iff } \quad \lim _{\theta \rightarrow 0+} \frac{1}{\theta}\left\|\gamma(\theta)-\gamma^{\prime}(\theta)\right\|_{\mathbf{L}^{1}}=0 \quad\left(\gamma, \gamma^{\prime} \in \Sigma_{u}\right) .
$$

Now assume that $u$ is piecewise Lipschitz, say with jumps at the points $x_{1}<\cdots<x_{N}$. The space of generalized tangent vectors at $u$ is then defined as $T_{u} \doteq \mathbf{L}^{1} \times \mathbb{R}^{N}$. To each $(v, \xi) \in T_{u}$, with $\xi=\left(\xi_{1}, \ldots, \xi_{N}\right)$, we associate the path $\gamma_{(v, \xi ; u)} \in \Sigma_{u}$ defined by

$\gamma_{(v, \xi ; u)}(\theta) \doteq u+\theta v+\sum_{\xi_{\alpha}<0}\left[u\left(x_{\alpha}^{+}\right)-u\left(x_{\alpha}^{-}\right)\right] \chi_{\left[x_{\alpha}+\theta \xi_{\alpha}, x_{\alpha}\right]}-\sum_{\xi_{\alpha}>0}\left[u\left(x_{\alpha}^{+}\right)-u\left(x_{\alpha}^{-}\right)\right] \chi_{\left[x_{\alpha}, x_{\alpha}+\theta \xi_{\alpha}\right]^{\circ}}$

More generally, we say that a path $\gamma \in \Sigma_{u}$ generates the generalized tangent vector $(v, \xi) \in T_{u}$, if $\gamma$ is equivalent to $\gamma_{(v, \xi ; u)}$, under the relation (21).

In other words, for small values of $\theta$, the function $u^{\theta} \doteq \gamma(\theta)$ can be obtained from $u$ by adding $\theta v$ and by shifting the positions of the jumps from $x_{\alpha}$ to $x_{\alpha}+\theta \xi_{\alpha}$. As $\theta \rightarrow 0+$, this 
procedure yields a first order approximation to $u^{\theta}$, with an error $o(\theta)$ in the $\mathbf{L}^{1}$ norm, with $o(\theta) / \theta \rightarrow 0$ as $\theta \rightarrow 0$. In connection with the above differential structure, one can define a kind of continuous differentiability property for maps $\gamma: \theta \mapsto u^{\theta} \in \mathbf{L}^{1}$, with piecewise Lipschitz values. Following [14], we say that a map $\gamma:] a, b\left[\mapsto \mathbf{L}^{1}\right.$ is a regular path if there exists an integer $N$ such that:

(i) Every function $u^{\theta} \doteq \gamma(\theta)$ is piecewise Lipschitz continuous with jumps at points $x_{1}^{\theta}<$ $\cdots<x_{N}^{\theta}$ continuously depending on $\theta$. Outside the jumps, each $u^{\theta}$ is continuous with a Lipschitz constant $L$ independent of $\theta$. All functions $u^{\theta}$ coincide outside some interval $[-M, M]$.

(ii) The map $\theta \mapsto u_{x}^{\theta}$ is continuous with values in $\mathbf{L}^{1}$.

(iii) There exists a continuous map $\theta \mapsto\left(v^{\theta}, \xi^{\theta}\right) \in \mathbf{L}^{1} \times \mathbb{R}^{N}$ such that for every $\theta$

$$
\lim _{\varepsilon \rightarrow 0+} \frac{1}{\varepsilon}\left\|\gamma(\theta+\varepsilon)-\gamma_{\left(v^{\theta}, \xi^{\theta} ; u^{\theta}\right)}(\varepsilon)\right\|_{\mathbf{L}^{1}}=0 .
$$

More generally, we say that a continuous map $\gamma:[a, b] \mapsto \mathbf{L}^{1}$ is a piecewise regular path if there exist points $a=\theta_{0}<\theta_{1}<\cdots<\theta_{\nu}=b$ such that the restriction of $\gamma$ to each open subinterval $] \theta_{j-1}, \theta_{j}[$ is a regular path.

Now consider any $u \in \mathcal{D}_{P L}$, say with jumps at $x_{1}<\cdots<x_{N}$. For every $\alpha=1, \ldots, N$, $i=1, \ldots, n$, let $\sigma_{\alpha}^{i}$ be the strength of the $i$-th wave in the Riemann problem at $x_{\alpha}$ Given a generalized tangent vector $(v, \xi) \in T_{u}=\mathbf{L}^{1} \times \mathbb{R}^{N}$, we define its weighted norm as

$$
\|(v, \xi)\|_{u} \doteq \sum_{\alpha=1}^{N} \sum_{i=1}^{n}\left|\sigma_{\alpha}^{i} \| \xi_{\alpha}\right| W_{i}^{u}\left(x_{\alpha}\right)+\sum_{i=1}^{n} \int_{-\infty}^{\infty}\left|v_{i}(x)\right| W_{i}^{u}(x) d x,
$$

Here $v_{i}(x) \doteq l_{i}(u(x)) \cdot v(x)$ is the $i$-th component of $v$, while $Q$ is the interaction potential defined at (3). The weight functions $W_{i}^{u}$ are defined by

$$
\begin{gathered}
W_{i}^{u}(x) \doteq 1+\kappa_{1} R_{i}^{u}(x)+\kappa_{1} \kappa_{2} Q(u), \\
R_{i}^{u}(x) \doteq \quad\left[\sum_{j \leq i} \int_{x}^{\infty}+\sum_{j \geq i} \int_{-\infty}^{x}\right]\left|u_{x}^{j}(y)\right| d y+\left[\sum_{\substack{k \leq i \\
x_{\alpha}>x}}+\sum_{\substack{k \geq i \\
x_{\alpha}<x}}\right]\left|\sigma_{\alpha}^{k}\right| \\
+\left\{\begin{array}{cc}
\sigma_{\beta}^{i} & \text { if } x=x_{\beta} \\
0 & \text { otherwise, }
\end{array}\right.
\end{gathered}
$$

for suitably large constants $\kappa_{1}, \kappa_{2}$. Intuitively, $R_{i}^{u}(x)$ can be regarded as the total strength of all waves in $u$ which approach an infinitesimal $i$-shock located at $x$. Finally, let $\gamma: \theta \mapsto u^{\theta}$ be a piecewise regular path defined on $[a, b]$, and let $\left(v^{\theta}, \xi^{\theta}\right)$ be its generalized tangent vector at $u^{\theta}$. In analogy with (14), we define the weighted length of $\gamma$ as

$$
\|\gamma\|_{*} \dot{=} \int_{a}^{b}\left\|\left(v^{\theta}, \xi^{\theta}\right)\right\|_{u^{\theta}} d \theta .
$$

The formula (15) now defines a weighted distance $d_{*}$ on $\mathcal{D}_{P L}$. Due to the choice of the weights in (25), it is not difficult to check that $d_{*}$ is uniformly equivalent to the standard $\mathbf{L}^{1}$ distance. By continuity, it can thus be extended to the whole domain $\mathcal{D}$. 


\section{Construction of the semigroup}

In the previous section we defined a weighted distance $d_{*}$ on the domain $\mathcal{D}$ in (13), uniformly equivalent to the $\mathbf{L}^{1}$ distance. At this stage, we are still a long way from proving that $d_{*}$ is contractive w.r.t. a semigroup generated by the system (1). Indeed, the very existence of the semigroup still needs to be established.

The main clue that $d_{*}$ may be contractive for the flow generated by the conservation laws is the fact that (A1) holds. More precisely, let $u=u(t, x)$ be a weak solution of (1), with $u(t, \cdot) \in \mathcal{D}_{P L}$ for every $t$. Assume that all shocks in $u$ are entropy-admissible and interect at most two at a time. Then, in analogy with (16), the generalized tangent vectors $(v(t, \cdot), \xi(t)) \in T_{u(t)}$ satisfy a linearized evolution equation, say

$$
\frac{d}{d t}(v, \xi)=\Lambda(u(t)) \cdot(v, \xi)
$$

The precise form of $\Lambda$ and the restarting conditions at times of shock interactions were derived in [14].

In this setting, for a suitable choice of the constants $\delta_{0}, \kappa_{i}$ in (4) and (25), the main result in [6] states that the map

$$
t \mapsto\|(v(t), \xi(t))\|_{u(t)}
$$

is a non-increasing function of time, also in the presence of shock interactions.

An immediate consequence is the following. Let $\gamma: \theta \mapsto \bar{u}^{\theta}$ be a regular path of initial data. Assume that, for each $\theta \in[a, b]$, the solution $u^{\theta}$ of the corresponding Cauchy problem remains regular (i.e., piecewise Lipschitz). Then the weighted length of the path $S_{t} \gamma: \theta \mapsto$ $u^{\theta}(t)$ is a non-increasing function of time.

Exploiting the above results, we now try to construct a semigroup generated by (1). A naive attempt goes as follows. Start with a dense family of sufficiently regular solutions, say piecewise Lipschitz. For any two solutions $u, w$, and $\varepsilon>0$, consider a regular path $\gamma_{0}$ joining $u(0)$ with $w(0)$, such that

$$
\left\|\gamma_{0}\right\|_{*} \leq d_{*}(u(0), w(0))+\varepsilon .
$$

Let $t \mapsto u^{\theta}(t)$ be the solution of (1) with initial data $u^{\theta}(0)=\gamma_{0}(\theta)$. For any $\tau>0$, consider the path $\gamma_{\tau}: \theta \mapsto u^{\theta}(\tau)$. We then have

$$
d_{*}(u(\tau), w(\tau)) \leq\left\|\gamma_{\tau}\right\|_{*} \leq\left\|\gamma_{0}\right\|_{*} \leq d_{*}(u(0), w(0)) .
$$

Since $\varepsilon$ is arbitrary, this shows that the flow of regular solutions is contractive w.r.t. the weighted distance $d_{*}$. This flow can thus be extended by continuity to a unique semigroup $S$ defined on the whole domain $\mathcal{D}$.

This approach was carried out in [4], in connection with systems whose characteristic fields are linearly degenerate. In the general case, a major technical difficulty arises. Indeed, piecewise Lipschitz initial data may lose their regularity in two ways:

- The number of jumps becomes infinite in finite time, due to repeated shock interactions.

- The Lipschitz constant becomes infinite, due to genuine nonlinearity.

If any one of the solutions $u^{\theta}$ loses its regularity at some time $t>0$, then for all $\tau>t$ the length of $\gamma_{\tau}$ can no longer be computed by (14), and the whole estimate breaks down. Still, the construction of the semigroup can be accomplished by means of two techniques. 
1. The system of conservation laws (1) is approximated by an auxiliary evolution equation, not necessarily in conservation form. In particular, for the new system, shock and rarefaction curves will locally coincide.

2. Given a family of solutions $u^{\theta}$, at the first time $\tau_{1}$ when one or more of these functions loses its regularity, a restarting procedure is performed. The path $\gamma_{\tau_{1}}: \theta \mapsto u^{\theta}\left(\tau_{1}\right)$ is thus replaced by a new regular path $\gamma_{\tau_{1}+}$, close to the old one and with almost the same length. The corresponding solutions will remain regular up to a time $\tau_{2}>\tau_{1}$ when a second restarting is performed, etc... In a finite number of steps, a path of approximate solutions is constructed on any given interval $[0, T]$. The basic estimate $(30)$ can still be recovered.

The first approximation technique was introduced in [9]. Alone, it suffices to handle the case of $2 \times 2$ systems. The restarting procedure was first used in the paper [11], to which we refer for all details.

Remarks. When $B$ is a hyperaccretive operator [18], the contractive semigroup generated by (11) can be obtained as limit of the approximating flows

$$
\frac{d u}{d t}+B_{\lambda}(u)=0
$$

For each $\lambda>0$, the Lipschitz continuous operator $B_{\lambda}$ is here defined as

$$
B_{\lambda} \doteq \lambda^{-1}\left(I-R_{\lambda}\right), \quad R_{\lambda} \doteq(I+\lambda B)^{-1} .
$$

On the other hand, for semigroups which are contractive w.r.t. a Riemann-type distance $d_{*}$, no general approximation procedure is known.

An alternative attempt to construct the semigroup generated by a system of conservation laws is to extend the formula (27). Namely, one can consider more general paths $\gamma: \theta \mapsto u^{\theta}$, with each $u^{\theta}$ not necessarily piecewise Lipschitz. A first step in this direction was taken in [12] by introducing a notion of shift differential, for paths taking values in the wider class of BV functions.

\section{Solutions with unbounded variation}

Theorem 1 states the well posedness of the Cauchy problem (1)-(2) within a set of functions $u \in \mathbf{L}^{1}$ with small total variation. In $[1,10]$, semigroups of solutions were constructed on domains $\mathcal{D}$ containing also functions with large variation.

A natural question is whether these flows can be extended to $\mathbf{L}^{\infty}$ functions, possibly with unbounded variation. This was accomplished in [3, 16] for special classes of $2 \times 2$ systems.

The following example, however, shows that such a continuous extension is not possible for general $n \times n$ systems.

Example 5. Consider the $3 \times 3$ strictly hyperbolic, linearly degenerate system

$$
\begin{aligned}
u_{t}+u_{x} & =0 \\
v_{t}-v_{x} & =0 \\
w_{t}+\left(\frac{1-u v}{6} \cdot w\right)_{x} & =0,
\end{aligned}
$$

with initial data

$$
u(0, x)=\varphi(x+1), \quad v(0, x)=\varphi(x-1), \quad w(0, x)=\eta(x),
$$


where $\eta$ is any smooth function and

$$
\varphi(x) \doteq\left\{\begin{array}{lll}
1 & \text { if } & 2^{-(2 n+1)}<|x|<2^{-2 n}, \\
-1 & \text { if } & 2^{-(2 n)}<|x|<2^{-2 n-1} .
\end{array}\right.
$$

Clearly we have

$$
u(t, x)=\varphi(x+1-t), \quad v(t, x)=\varphi(x-1+t) .
$$

To obtain the component $w$, call $s \mapsto y(s ; t, x)$ the characteristic line through the point $(t, x)$, i.e. the solution to the Cauchy problem

$$
\begin{aligned}
\dot{y}(s)=f(s, y(s)) & \doteq \frac{1-u(s, y) v(s, y)}{6}, \\
y(t) & =x,
\end{aligned}
$$

with $u, v$ given at (34). For $s, t \in[0,1[$ all these Cauchy problems have a unique solution and the values $y(s ; t, x)$ are well defined. Together with $(34)$, the function

$$
w^{(\eta)}(t, x) \doteq \frac{\partial y(0 ; t, x)}{\partial x} \cdot \eta(y(0 ; t, x))
$$

provides the unique solution to the Cauchy problem $(31)-(32)$, on the strip $[0,1[\times \mathbb{R}$.

Observe that the two functions

$$
y(s)=\frac{s-1}{3}, \quad y(s)=0
$$

are both solutions of $(35)$ with initial data $y(1)=0$. For any $\varepsilon>0$, applying the divergence theorem on the domain

$$
\Omega_{\varepsilon} \doteq\{(t, x) ; \quad t \in[0,1-\varepsilon], \quad(t-1) / 3 \leq x \leq 0\}
$$

we obtain

$$
\int_{-\varepsilon / 3}^{0} w(1-\varepsilon, x) d x=\int_{-1 / 3}^{0} \eta(x) d x \doteq \delta_{0} .
$$

If in (38) we have $\delta_{0} \neq 0$, then as $t \rightarrow 1$, the solution $w(t, \cdot)$ has no limit in $\mathbf{L}_{l o c}^{1}$. Its weak limit is a measure having a mass $\delta_{0}$ concentrated at the origin. We now show that the solution can be prolonged to the strip $[0,2] \times \mathbb{R}$ in infinitely many different ways. Indeed, the components $u, v$ are always given by (34). To obtain $w$ for $t \in[1,2]$, we first choose any smooth positive function $\eta^{\prime}$ such that

$$
\eta^{\prime}(x)=\eta(x) \quad \text { if } \quad x \notin[-1 / 3,0], \quad \int_{-1 / 3}^{0} \eta^{\prime}(x) d x=\delta_{0} .
$$

Calling $w^{\eta^{\prime}}$ the corresponding solution of (1) defined for $t<1$, we can now define

$$
w^{\eta, \eta^{\prime}}(t, x) \doteq \begin{cases}w^{\eta}(t, x) & \text { if } \quad t<1, \\ w^{\eta^{\prime}}(2-t,-x) & \text { if } \quad 1<t \leq 2 .\end{cases}
$$

Using the relations (39), since all characteristics starting at time $t=0$ within the interval $[-1 / 3,0]$ meet at the point $(\bar{t}, \bar{x})=(1,0)$, one checks that the function $w^{\eta, \eta^{\prime}}$ in $(40)$ provides a solution to the Cauchy problem (31)-(32). Clearly, $w^{\eta, \eta^{\prime}}(2, x)=\eta^{\prime}(-x)$. Different choices of $\eta^{\prime}$ thus yield infinitely many distinct weak solutions. 


\section{Proof of Lemma 1}

As a preliminary, observe that the integrand

$$
\phi(t) \doteq \liminf _{h \rightarrow 0+} \frac{\left\|w(t+h)-S_{h} w(t)\right\|}{h}
$$

in (7) is measurable. Indeed, for every $h>0$, the function $f_{h}(t) \doteq\left\|w(t+h)-S_{h} w(t)\right\|$ is continuous. By continuity of the maps $h \mapsto f_{h}(t)$, we have

$$
\phi(t)=\lim _{\varepsilon \rightarrow 0+} \inf _{h \in \mathbf{Q} \cap] 0, \varepsilon]} f_{h}(t),
$$

where the infimum is taken only over rational values of $h$. Hence $\phi$ is Borel measurable.

Next, let $\varepsilon>0$ be given. To fix the ideas, let $M>L$ be a Lipschitz constant for $w$, so that

$$
\|w(t)-w(s)\| \leq M|t-s|, \quad t, s \in[0, T] .
$$

By Lusin's theorem, there exists a compact set $J \subset[0, T]$ and a continuous function $\psi$ such that

$$
\operatorname{meas}(J)>T-\varepsilon, \quad \int_{0}^{T}|\psi(s)-\phi(s)| d s<\varepsilon, \quad \psi(x)=\phi(x) \text { for all } x \in J .
$$

For $t \in[0, T]$, define

$$
\begin{aligned}
\Psi_{\varepsilon}(t) & \doteq L \cdot \int_{0}^{t}[\varepsilon+\psi(s)] d s+2 M L \cdot \operatorname{meas}([0, t] \backslash J), \\
\tau & \doteq \sup \left\{t \in[0, T] ; \quad\left\|S_{T-t} w(t)-S_{T} w(0)\right\| \leq \Psi_{\varepsilon}(t)\right\} .
\end{aligned}
$$

We claim that $\tau=T$. Indeed, assume the contrary. By continuity, at $t=\tau$ we have

$$
\left\|S_{T-\tau} w(\tau)-S_{T} w(0)\right\|=\Psi_{\varepsilon}(\tau)
$$

If $\tau \notin J$, since $J$ is closed we can choose $h>0$ such that $[\tau, \tau+h] \cap J=\emptyset$. By (44) and (41) this implies

$$
\begin{aligned}
\left\|S_{T-\tau-h} w(\tau+h)-S_{T} w(0)\right\| & \leq\left\|S_{T-\tau} w(\tau)-S_{T} w(0)\right\|+L \cdot\left\|w(\tau+h)-S_{h} w(\tau)\right\| \\
& \leq \Psi_{\varepsilon}(\tau)+L \cdot 2 M h \\
& \leq \Psi_{\varepsilon}(\tau+h) .
\end{aligned}
$$

In the case $\tau \in J$, by (42) and the continuity of $\psi$, there exists $h>0$ such that

$$
\frac{\left\|w(\tau+h)-S_{h} w(\tau)\right\|}{h} \leq \psi(\tau)+\frac{\varepsilon}{2} \leq \min _{s \in[\tau, \tau+h]} \psi(s)+\varepsilon .
$$

By (44), this yields

$$
\begin{aligned}
\left\|S_{T-\tau-h} w(\tau+h)-S_{T} w(0)\right\| & \leq\left\|S_{T-\tau} w(\tau)-S_{T} w(0)\right\|+L \cdot\left\|w(\tau+h)-S_{h} w(\tau)\right\| \\
& \leq \Psi_{\varepsilon}(\tau)+L \cdot \int_{\tau}^{\tau+h}[\psi(s)+\varepsilon] d s \\
& \leq \Psi_{\varepsilon}(\tau+h),
\end{aligned}
$$

again in contradiction with the maximality of $\tau$. Therefore, $\tau=T$. Recalling (42) and the definition of $\Psi_{\varepsilon}$, we deduce

$$
\begin{aligned}
\left\|w(T)-S_{T} w(0)\right\| & \leq \Psi_{\varepsilon}(T) \\
& =\int_{0}^{T} \psi(t) d t+L \varepsilon T+2 M L \cdot \operatorname{meas}([0, T] \backslash J) \\
& \leq L \cdot \int_{0}^{T} \phi(t) d t+L(\varepsilon+\varepsilon T)+2 M L \varepsilon .
\end{aligned}
$$

Since $\varepsilon>0$ is arbitrary, the lemma is proved. 


\section{Références}

[1] P. Baiti \& A. Bressan, The semigroup generated by a Temple class system with large data, Diff. Integ. Equat, to appear.

[2] P. Baiti \& H.K. Jenssen, On the front tracking algorithm, Preprint S.I.S.S.A., Trieste 1996.

[3] P. Batti \& H.K. Jenssen, Well posedness for a class of $2 \times 2$ conservation laws with $\mathbf{L}^{\infty}$ data, Preprint S.I.S.S.A., Trieste 1996.

[4] A. Bressan, Contractive metrics for nonlinear hyperbolic systems, Indiana Univ. Math. J. 37 (1988), 409-421.

[5] A. BRESSAN, Global solutions of systems of conservation laws by wave-front tracking, J. Math. Anal. Appl. 170 (1992), 414-432.

[6] A. Bressan, A locally contractive metric for systems of conservation laws, Ann. Scuola Norm. Sup. Pisa IV-22 (1995), 109-135.

[7] A. Bressan, The unique limit of the Glimm scheme, Arch. Rational Mech. Anal. 130 (1995), 205-230.

[8] A. Bressan \& G. Colombo, Existence and continuous dependence for discontinuous O.D.E.'s, Boll. Unione Matem. Italiana 4-B (1990), 295-311.

[9] A. Bressan \& R.M. Colombo, The semigroup generated by $2 \times 2$ systems of conservation laws, Arch. Rational Mech. Anal. 133 (1995), 1-75.

[10] A. Bressan \& R.M. Colombo, Unique solutions of $2 \times 2$ conservation laws with large data, Indiana Univ. Math. J. 44 (1995), 677-725.

[11] A. Bressan, G. Crasta \& B. Piccoli, Well-posedness of the Cauchy problem for $n \times n$ systems of conservation laws, preprint S.I.S.S.A., Trieste, 1996 .

[12] A. Bressan \& G. Guerra, Shift-differentiability of the flow generated by a conservation law, Discrete and Continuous Dynamical Systems 3 (1997), 35-58.

[13] A. Bressan \& P. LeFloch, Uniqueness of weak solutions to systems of conservation laws, Arch. Rational Mech. Anal, to appear.

[14] A. BREssan \& A. Marson, A variational calculus for discontinuous solutions of systems of conservation laws, Comm. Part. Diff. Equat. 20 (1995), 1491-1552.

[15] A. Bressan \& A. Marson, Error bounds for a deterministic version of the Glimm scheme, Arch. Rational Mech. Anal., to appear.

[16] A. Bressan \& W. Shen, Uniqueness for discontinuous O.D.E. and conservation laws, Nonlinear Analysis, to appear.

[17] M. Crandall, The semigroup approach to first-order quasilinear equations in several space variables, Israel J. Math. 12 (1972), 108-132.

[18] K. Deimling, Nonlinear Functional Analysis, Springer-Verlag, Berlin, 1985.

[19] R. DiPerna, Global existence of solutions to nonlinear hyperbolic systems of conservation laws, J. Differential Equations 20 (1976), 187-212.

[20] J. GLimm, Solutions in the large for nonlinear hyperbolic systems of equations, Comm. Pure Appl. Math. 18 (1965), 697-715.

[21] F. John, Formation of singularities in one-dimensional wave propagation, Comm. Pure Appl. Math. 27 (1974), 377-405.

[22] S. Kruzkov, First order quasilinear equations with several space variables, Math. U.S.S.R. Sbornik 10 (1970), 217-243. 
[23] P. Lax, Hyperbolic systems of conservation laws II, Comm. Pure Appl. Math. 10 (1957), $537-566$.

[24] T.P. Liu, The deterministic version of the Glimm scheme, Comm. Math. Phys. 57 (1977), 135-148.

[25] O.A. Oleinik, Discontinuous solutions of nonlinear differential equations, Usp. Mat. Nauk 12 (1957), 3-73. English Transl. Amer. Math. Soc. Transl. Ser. 2, Vol.26, 95-172.

[26] N.H. Risebro, A front-tracking alternative to the random choice method, Proc. Amer. Math. Soc. 117 (1993), 1125-1139.

[27] B. Rozdestvenskit \& N. Yanenko, Systems of Quasi-Linear Equations, A. M. S. Translations of Mathematical Monographs, Vol. 55, 1983.

[28] J. Smoller, Shock waves and reaction-diffusion equations, Second Edition, SpringerVerlag, New York, 1994.

ESAIM: Proc., VoL. 3, 1998, 23-36 